\title{
The role of lipoprotein-associated phospholipase A2 in acute kidney injury of septic mice
}

\author{
Guiwen Liang ${ }^{1 \#}$, Ruo Wu ${ }^{1 \#}$, Lan Jiang ${ }^{2}$, Yanfang Liu ${ }^{3}$, Guomin Mao ${ }^{1}$, Zhongwei Huang ${ }^{1}$, Lei Qi ${ }^{1}$, \\ Haiyan Jiang ${ }^{1}$
}

${ }^{1}$ Department of Emergency Medicine, Affiliated Hospital of Nantong University, Nantong, China; ${ }^{2}$ Department of Gastroenterology, First People's Hospital of Zhangjiagang City, Zhangiiagang, China; ${ }^{3}$ Department of Anesthesiology, Affiliated Hospital of Nantong University, Nantong, China

Contributions: (I) Conception and design: L Qi, H Jiang; (II) Administrative support: Z Huang, G Liang; (III) Provision of study materials or patients: Y Liu, G Mao; (IV) Collection and assembly of data: L Jiang; (V) Data analysis and interpretation: R Wu; (VI) Manuscript writing: All authors; (VII) Final approval of manuscript: All authors.

\#These authors contributed equally to this work.

Correspondence to: Lei Qi; Haiyan Jiang. Department of Emergency Medicine, Affiliated Hospital of Nantong University, 20 West Temple Road, Nantong, China. Email: qilei723@ntu.edu.cn; jhy@ntu.edu.cn.

\begin{abstract}
Background: This experiment aimed to investigate the role and mechanism of lipoprotein-associated phospholipase A2 (Lp-PLA2) in kidney injury in septic mice induced by cecal ligation and perforation (CLP). Methods: Male BALB/c mice were randomly divided into two groups: sham-operation group (Sham group) and septic group (CLP group). The septic model was simulated by cecal ligation and puncture method, but only cecal ligation was used for the sham operation group. The whole serum and renal tissue samples of the mice were collected 24 hours after modeling in both groups. Hematoxylin-eosin (HE) staining was used to observe the pathological changes of renal tissue, the renal injury score was recorded, and the creatinine $(\mathrm{Cr})$ and blood urea nitrogen (BUN) levels were detected by automatic biochemical analyzer, while the serum LpPLA2 level was detected by enzyme-linked immunosorbent assay (ELISA). The 7-day survival rate and the survival curve of the two groups were statistically analyzed.

Results: Compared with the Sham group, the pathological score of renal injury in the CLP Group was higher, the level of Lp-PLA2 in serum was significantly increased (all $\mathrm{P}<0.01$ ), and the expression of LpPLA2 in renal tissue was significantly elevated (all $\mathrm{P}<0.01$ ). Furthermore, the 7 -day survival rate of the Sham group was $90 \%$, while that of CLP group was $25 \%$.

Conclusions: The expression level of Lp-PLA2 in blood and kidney tissue of septic mice was increased and correlated with prognosis. However, the predictive value of Lp-PLA2 for prognosis in septic mice needs further study.
\end{abstract}

Keywords: Lp-PLA2; sepsis; acute kidney injury (AKI); inflammation

Submitted Jul 23, 2020. Accepted for publication Oct 09, 2020.

doi: $10.21037 /$ tau-20-1173

View this article at: http://dx.doi.org/10.21037/tau-20-1173

\section{Introduction}

Sepsis is a systemic inflammatory response syndrome (SIRS) caused by infection (1), which is one of the important causes of death in intensive care unit (ICU) patients. The prevalence and the mortality rate of sepsis are high, and, according to statistics, about 30 million people are infected with sepsis and 6 million people die of sepsis every year (2). The pathogenesis of sepsis is related to the imbalance of inflammatory response and immune regulation (3). Microorganisms break through the skin barrier and invade the blood flow, causing an active host defense response, stimulating phagocytes, recruiting inflammatory cells, and leading to the release of inflammatory mediators and the 
occurrence of inflammatory cascade reaction. This in turn results in the enhancement of systemic inflammation (4), eventually precipitating organ dysfunction or even death. The kidney is a common organ involved in sepsis, prone to develop into acute kidney injury (AKI). AKI is one of the most common and serious complications of sepsis. According to statistics, about $50 \%$ of septic patients have AKI, and one-third of them die of this condition (5). The pathogenesis and pathophysiological mechanism of AKI in sepsis are complex and multifactorial. AKI pathogenesis is related to renal ischemia-reperfusion injury, inflammatory factors, nitric oxide theory, apoptosis theory, endothelial dysfunction, endothelin (ET) effect, endotoxin direct damage, oxidative stress injury, and other factors (6). Meanwhile, AKI pathophysiology is related to the changes in the renal hemodynamics, endothelial dysfunction, infiltration of inflammatory cells in the renal parenchyma, thrombosis in glomeruli, obstruction of tubules, necrotic cells, and debris (7). A study by Federspiel et al. (8) involving patients with sepsis-associated AKI found that prolonged AKI for more than 7 days was associated with ICU stay, ventilator use, and cardiovascular failure, resulting in low prognosis, a high recurrence rate, and a high mortality rate. Therefore, it is crucial to actively identify biomarkers and therapeutic targets that can identify sepsisrelated AKI early, so as to enable early prevention and intervention, thereby reducing the incidence and mortality. Phospholipase A2 (PLA2) is an enzyme family that participates in lipid metabolism, hydrolyzes phospholipids, and releases arachidonic acid and lysophosphatidylcholine (lysoPC) (9). It is found widely in bacteria, mammalian cells, and secretions (10). According to the location, amino acid sequence homology, and biochemical characteristics, PLA2 family can be divided into three types: Ca2+dependent, comprising (I) secretory (sPLA2, including PLA2-I, II, III, V, IX, and X) and (II) cytoplasmic types (cPLA2, including PLA2-IV); and (III) $\mathrm{Ca}^{2+}$-independent (iPLA2, including PLA2-VI, VII, and VIII) (11). It plays an important role in the inflammatory response by triggering the production of inflammatory mediators and participates in a variety of acute and chronic inflammatory diseases. In recent years, several studies have shown that PLA2 is involved in the pathogenesis of acute respiratory syndrome (ARDS) (12), acute pancreatitis (13), sepsis (14), and has great value in the diagnosis of inflammatory diseases. Lipoprotein-associated phospholipase A2 (Lp-PLA2), a member of the phospholipase A2 superfamily, is a $\mathrm{Ca}^{2+}-$ independent secreted PLA2 (15). Because of its activity in degrading platelet-activating factor (PAF), also known as platelet-activating factor acetylhydrolase (PAF-AH) (16), it is synthesized and secreted by inflammatory cells such as macrophages, $\mathrm{T}$ cells, and mast cells in the intima of blood vessels. It can hydrolyze oxidized phospholipids with proinflammatory properties, release lysoPC, and form nonesterified fatty acids (oxNEFA) (primary arachidonic acid) (17-19). LysoPC and oxidized non-esterified fatty acids are pro-inflammatory mediators that stimulate the production of adhesion factors and cytokines, and form monocyte chemotaxis, which could be turned into macrophages after endometrial aggregation (20). At the same time, they can cause endothelial dysfunction and induce endothelial cells to express leukocyte adhesion factor, platelet-derived growth factor, and epidermal growth factor (21), which jointly participate in the inflammatory reaction and aggravate the vicious circle of cascade reaction. Although Lp-PLA2 can degrade the platelet-activating factor (PAF) and play an anti-inflammatory and antithrombotic role, this is insignificant compared to the pro-inflammatory effect (20). Results of related studies suggest that Lp-PLA2 has potential value as a biomarker of inflammatory diseases. A fair amount of literature attests to its upregulation in coronary heart disease (22), stroke (23), pancreatitis (24), and myocarditis (25), but the role in AKI caused by sepsis has not been studied in detail. In the past, a large number of studies related to sepsis induced acute kidney injury, but the majority of clinical related studies. The potential biomarkers for its prevention and treatment are rarely studied. However, according to our previous clinical experiments, we found that there is a certain correlation between Lp-PLA2 and sepsis related renal injury, so we conducted further research. Thus, the purpose of the present study was to clarify the role of Lp-PLA2 in renal injury in septic mice and its related mechanism, and to provide the theoretical basis for early clinical detection and treatment of AKI caused by sepsis.

We present the following article in accordance with the ARRIVE reporting checklist (available at http://dx.doi. org/10.21037/tau-20-1173).

\section{Methods}

\section{Animal grouping and establishment of a sepsis model}

Male BALB/c mice aged 6-8 weeks (Kay Biological Technology, Shanghai, China) were randomly divided into two groups, the sham operation group (Sham group) 
and the sepsis group (CLP Group). In brief, the model of CLP group was established by cecal ligation and puncture combined with an experimental method described elsewhere (26). The mice were anesthetized (3\% chloral hydrate $1 \mathrm{~mL} / 100 \mathrm{~g}$ ) to the appropriate depth. The mice were fixed on the experimental operating table in a supine position. The skin was prepared and the operation area was disinfected. A $1-\mathrm{cm}$ incision was made on the midline of the abdomen. The end of the cecum was ligated with No. 4 sterile silk thread at the position of $1-1.5 \mathrm{~cm}$. The cecal end was punctured and extruded with a No. 20 needle. A small amount of feces was disinfected with iodophor cotton swab and returned to the abdominal cavity with sterile tweezers. Finally, the abdomen was closed by suture layer by layer. After that, $30 \mathrm{~mL} / \mathrm{kg}$ body weight of warm-up normal saline was injected intraperitoneally for fluid resuscitation (27). After the operation, the mice were free to eat and drink. In the Sham group, the abdominal cavity was opened as in the CLP Group, and cecal ligation was performed without further cecal puncture. Experiments were performed under a project license (No.: 2018-L062) granted by the Ethics Committee of the Affiliated Hospital of Nantong University, in compliance with GSPC (Gold standard publication checklist) for the care and use of animals.

\section{Animal sampling}

The mice in the two groups were anesthetized at an appropriate depth $24 \mathrm{~h}$ after the operation. The abdominal cavity was opened along the midline of the abdomen. Whole blood $800-1,000 \mu \mathrm{L}$ was collected from each mouse through the heart and centrifuged at $1,000 \mathrm{R} / \mathrm{min}$ for $10 \mathrm{~min}$ after standing at room temperature for $1 \mathrm{~h}$. The supernatant was put into a $1.5-\mathrm{mL}$ Eppendorf tube and stored in a $-80{ }^{\circ} \mathrm{C}$ refrigerator for subsequent detection. After that, the exposed aorta and kidney were separated, the abdominal aorta was cutoff, and blood was completely drawn. The bilateral kidney tissues were stored in a $1.5-\mathrm{mL}$ Eppendorf tube and frozen in a $-80{ }^{\circ} \mathrm{C}$ refrigerator for subsequent detection.

\section{Hematoxylin-eosin (HE) staining of renal tissue}

One side of the renal tissue was fixed with $4 \%$ paraformaldehyde for $24 \mathrm{~h}$, paraffin-embedded, sliced, and then stained with hematoxylin and eosin (HE; Sigma) (28). Under a Leica DM 4000B microscope (Leica Microsystems, Wetzlar, Germany), 5-10 visual fields were selected to observe the pathological changes of renal tissue. The tubular injury was defined as tubular epithelial swelling, brush edge loss, vacuolar degeneration, necrotic tubules, cast formation, and desquamation. Scoring criteria were the following: 0 , normal tissue; 1 , damaged area of renal tubules $<25 \%$; 2 , damaged area of renal tubules between $25 \%$ and $50 \%$; 3, damaged area of renal tubules between $50 \%$ and $75 \% ; 4$, damaged area of renal tubules between $75 \%$ and $100 \%$.

\section{Creatinine (Cr) and blood urea nitrogen (BUN) levels}

The levels of $\mathrm{Cr}$ and BUN in serum were analyzed by an automatic biochemical analyzer (Roche Diagnostic, Shanghai). The parameters were set according to the instructions of the instrument, the samples and reagents to be tested were placed, and the data were read.

\section{The levels of Lp-PLA2 in serum and kidney were measured by enzyme-linked immunosorbent assay (ELISA)}

The serum and kidney tissues stored in $-80{ }^{\circ} \mathrm{C}$ refrigerator were taken out for thawing, and then the operation was carried out in strict accordance with the instructions of the (Lp-PLA2) ELISA Kit (R \& D Systems, Minneapolis, MN, USA), and the level of Lp-PLA2 in kidney tissue was determined.

\section{Seven-day survival rate of mice}

Twenty male BALB/c mice aged 6-8 weeks (Kay Biological Technology, Shanghai, China) were randomly divided into two groups: the Sham group and the CLP Group. After the establishment of the CLP model, mice in each group were first observed for 7 days of survival. The deaths were observed and recorded each day for 7 days, and the survival curves were drawn and analyzed statistically.

\section{Statistical methods}

GraphPad Prism v.8 (Graphpad Software, San Diego, CA, USA) was used for all statistical analyses. The significant differences concerning the interaction effects were analyzed using one-way analysis of variance (ANOVA) and Tukey's test. The normal distribution of measurement data are expressed as mean \pm standard error $(\bar{x} \pm s)$, and the comparison between the two groups was conducted with a two-tailed Student's $t$-test. A Kaplan-Meier survival curve 
was used to analyze survival. A P value $<0.05$ was considered statistically significant.

\section{Results}

\section{Histopathological observation of kidneys}

In the Sham group, the structure of kidney tissue was clear, the renal tubules and glomeruli were normal; no degeneration, atrophy, swelling, necrosis, or inflammatory infiltration was found in renal tubular epithelial cells, and the lumen was not dilated. In the CLP Group, the renal tissue was edematous, the glomerular structure was not clear, and a small amount of fibrous tissue hyperplasia was accompanied with a large degree of inflammatory cell infiltration; the capillary lumen became narrow or even

Table 1 The renal histopathological scores of the Sham group $(\mathrm{n}=10)$ and CLP group $(\mathrm{n}=10) 24 \mathrm{~h}$ after operation

\begin{tabular}{lc}
\hline Group & 24-h renal histopathological scores \\
\hline Sham & $0.89 \pm 0.20$ \\
CLP & $1.96 \pm 0.40$ \\
\hline
\end{tabular}

CLP, cecal ligation and perforation.

A

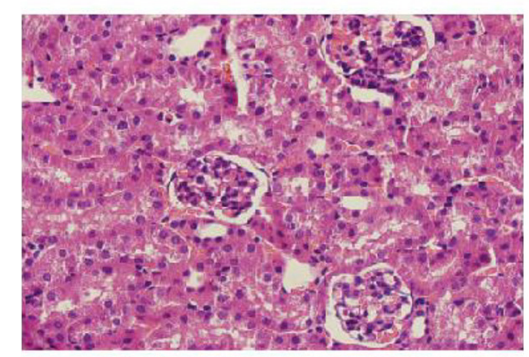

B

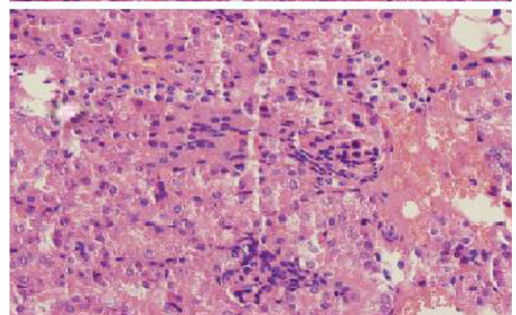

occluded, and the renal small cystic cavity was significantly narrowed; compared with the Sham group, the renal injury score was significantly increased $(\mathrm{P}<0.05)$ (Table 1). Compared with the normal kidney tissue, there was no significant difference in the pathological score of renal tissue injury in the Sham group; the pathological score of renal tissue injury in the CLP Group was significantly increased at each time point after the operation, and there was a significant difference compared with the Sham group $(\mathrm{P}<0.05)$ (Figure 1).

\section{Serum levels of Cr and BUN}

The levels of $\mathrm{Cr}$ and BUN in the serum of the Sham group were within the normal physiological range, while those in the CLP Group were significantly higher than those in the Sham group (all $\mathrm{P}<0.05)$ (Table 2; Figure 2).

\section{Changes in Lp-PLA2 level in serum}

The serum Lp-PLA2 level in the Sham group was lower than that in the CLP-induced septic mice, and the difference was statistically significant (all $\mathrm{P}<0.05$ ) (Table 3; Figure 3).

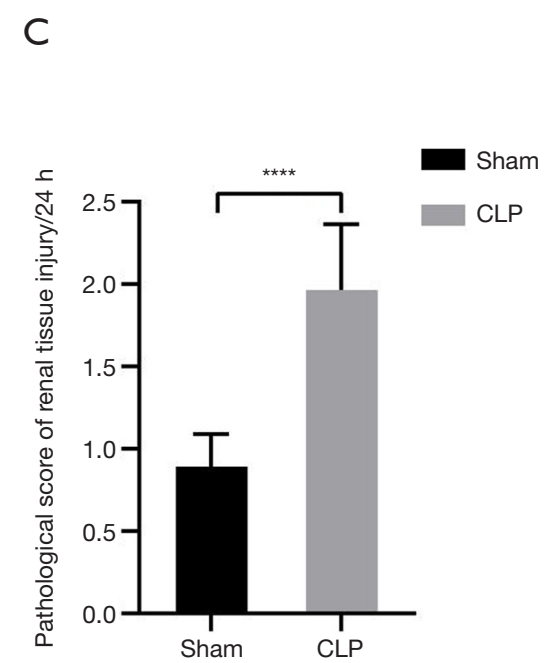

Figure 1 Renal tissue injury in the CLP group was more severe than that in Sham group 24 hours after the operation. (A) By HE staining, the pathological structure of the kidney in the Sham group was observed under the microscope (200 times magnification); (B) By HE staining, the pathological structure of the kidney in the CLP group was observed $24 \mathrm{~h}$ after the operation (200 times magnification); (C) the renal histopathological score of the CLP group $(n=10)$ was significantly higher than that of the Sham group ( $n=10)$. The results showed a significant difference. ${ }^{* * *}, \mathrm{P}<0.001$. CLP, cecal ligation and perforation. 


\section{Changes in Lp-PLA2 level in renal tissue}

The level of Lp-PLA2 in the Sham group was lower than that in the CLP-induced septic mice, and the difference was statistically significant (all $\mathrm{P}<0.05)($ Table 3; Figure 3).

\section{Seven-day survival rate}

The 7-day survival rate of the CLP Group was significantly lower than that of the Sham group $(25.0 \%$ vs. $90.00 \%$, $\mathrm{P}<0.001$ ) (Figure 4).

\section{Discussion}

Sepsis can lead to multiple organ dysfunction syndrome, which seriously affects the prognosis of ICU patients. The mortality of sepsis complicated with acute renal failure has been reported to be as high as $74.5 \%$, while that without acute renal failure was reported to be $45.2 \%$ (29). Indepth study on the pathogenesis of sepsis-induced AKI has revealed that inflammatory necrosis of renal tubular

Table $2 \mathrm{Cr}$ and BUN levels in the serum of the CLP group ( $\mathrm{n}=10)$ and Sham group $(\mathrm{n}=10)$ mice

\begin{tabular}{lcc}
\hline Group & Creatinine $(\mu \mathrm{mol} / \mathrm{L})$ & Blood urea nitrogen $(\mu \mathrm{mol} / \mathrm{L})$ \\
\hline Sham & $75.28 \pm 1.294$ & $7.735 \pm 0.3751$ \\
CLP & $121.5 \pm 2.088$ & $12.87 \pm 0.4683$ \\
\hline
\end{tabular}

$\mathrm{Cr}$, creatinine; BUN, blood urea nitrogen; CLP, cecal ligation and perforation. epithelial cells may play a key role in this process. The results of the present study showed the necrosis of renal tissue in the CLP group to be significantly higher than that in the Sham group. Meanwhile, the experimental results also indicated that inflammatory lesions in renal tissue could lead to changes in the renal vascular bed, while promoting inflammatory cell infiltration and thrombosis. Lp-PLA2, also known as platelet-activating factor phthalide hydrolase (PAF-AH), is an inflammatory marker closely related to atherosclerosis and ischemic cardiovascular and cerebrovascular diseases. It can catalyze the hydrolysis of lipoproteins and phosphatidylglycerol bonds on the cell membrane to form uncooled fatty acids and lysophospholipids. Lp-PLA2 is a serine lipase composed of 441 amino acid residues with a relative molecular weight of $45.4 \mathrm{KD}$ (30); it mainly exists in blood vessels and is produced by inflammatory cells and regulated by inflammatory mediators. Compared with the Sham group, severe necrosis, edema, infiltration of inflammatory cells, unclear glomerular structure, and loss of original physiological structure occurred in the Sepsis model group. Meanwhile, through the detection of serum $\mathrm{Cr}$, serum urea nitrogen value was used to verify the degree of renal injury and necrosis. Furthermore, the levels of Lp-PLA2 in the kidney and serum of the Sham and CLP groups were detected by ELISA. This indicates that, with the further development of sepsis, severe systemic infection followed by septic shock will occur, which in turn results in a low kidney blood flow and poor renal perfusion. The aggravation of the disease leads to the release of vascular inflammatory factors.
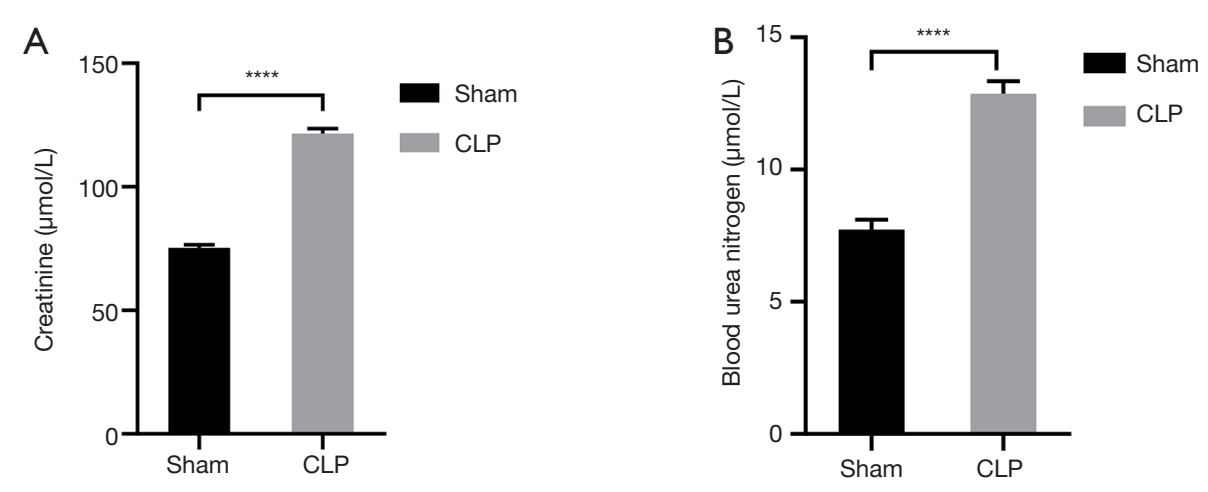

Figure 2 The $\mathrm{Cr}$ and BUN in the CLP group ( $\mathrm{n}=10)$ were higher than those in the Sham group. An automatic biochemical analyzer (Roche diagnostic, Shanghai) was used to evaluate the Cr value of mice. (A) The Cr in the CLP group (n=10) was significantly higher than that in the Sham group, and exceeded the normal range; (B) the BUN level in the CLP group $(n=10)$ was significantly higher than that in the Sham group and exceeded the normal range. The results showed a significant difference. ${ }^{* * * *}, \mathrm{P}<0.001$. Cr, creatinine; BUN, blood urea nitrogen; CLP, cecal ligation and perforation. 
Table 3 Expression of Lp-PLA2 in the serum and kidney of CLP group $(\mathrm{n}=10)$ and Sham group $(\mathrm{n}=10)$ mice

\begin{tabular}{lcc}
\hline Group & $\begin{array}{c}\text { Serum LP-PLA2 } \\
(\mathrm{ng} / \mathrm{mL})\end{array}$ & $\begin{array}{c}\text { Kidney tissue LP-PLA2 } \\
(\mathrm{ng} / \mathrm{mL})\end{array}$ \\
\hline Sham & $77.94 \pm 2.329$ & $79.87 \pm 2.719$ \\
CLP & $92.43 \pm 1.866$ & $92.14 \pm 1.544$ \\
\hline
\end{tabular}

CLP, cecal ligation and perforation.

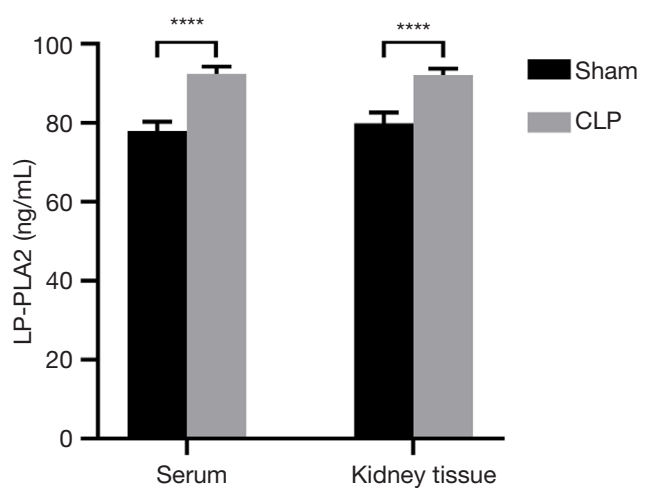

Figure 3 Lp-PLA2 was expressed in the serum and kidney of the CLP group ( $n=10)$ and Sham group $(n=10)$. The expression levels of Lp-PLA in the serum and kidney of the CLP group $(n=10)$ were higher than those in the Sham group $(n=10)$. ${ }^{* * *}, \mathrm{P}<0.001$. CLP, cecal ligation and perforation.

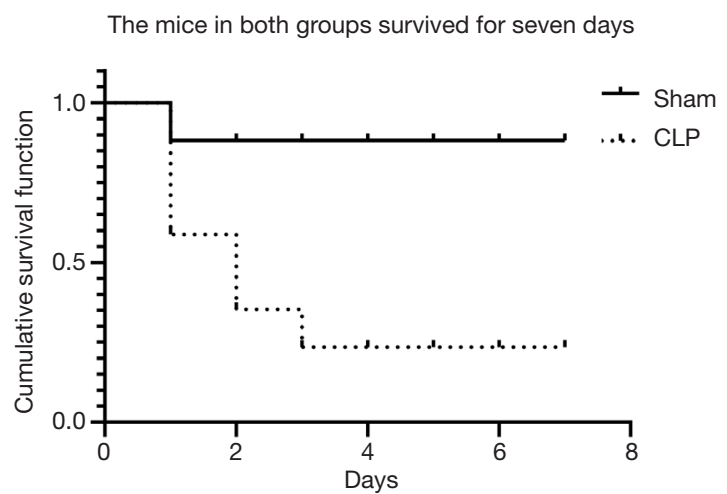

Figure 4 The survival rate of the CLP group (n=20) was compared with that of the Sham group $(n=20)$. Deaths occurred in the Sham group on the first day, but did not occur again. The mortality of CLP group mice was much higher than that of Sham group mice on the first day, second day, and third day, indicating that the prognosis of the CLP group was poor. CLP, cecal ligation and perforation.
As an enzyme mediated by vascular inflammatory factors, Lp-PLA2 also increases significantly. Lp-PLA2 hydrolyzes oxidized phosphatidylcholine on low-density lipoprotein (LDL) on arterial intima to produce pro-inflammatory mediators such as lysoPC and free oxidized fatty acids. It can stimulate the production of adhesion factors and cytokines, and promote monocytes to accumulate from the lumen to intima. These monocytes then develop into macrophages causing oxidative activation, which aggravates the damage of renal vessels. Moreover, activated macrophages and apoptotic cells will produce and release more Lp-PLA2 into the blood, forming an injurious cycle. Some studies have shown that (31) Lp-PLA2 activity is positively correlated with the concentration in blood, which eventually leads to the further development of inflammation and aggravates the process of sepsis.

\section{Conclusions}

The results of our study show that the expression of LpPLA2 in blood and kidney tissue of septic mice was increased, and the up-regulation of Lp-PLA2 is positively correlated with the severity of acute kidney injury induced by sepsis, which is expected to become a diagnostic and prognostic indicator for acute kidney injury induced by sepsis.

\section{Acknowledgments}

Funding: This study was supported by the National Natural Science Foundation of China Youth (81801893) and the Nantong Clinical Medicine Research Center (HS2019005).

\section{Footnote}

Reporting Checklist: The authors have completed the ARRIVE reporting checklist. Available at http://dx.doi. org/10.21037/tau-20-1173

Data Sharing Statement: Available at http://dx.doi. org/10.21037/tau-20-1173

Conflicts of Interest: All authors have completed the ICMJE uniform disclosure form (available at http://dx.doi. org/10.21037/tau-20-1173). The authors have no conflicts 
of interest to declare.

Ethical Statement: The authors are accountable for all aspects of the work in ensuring that questions related to the accuracy or integrity of any part of the work are appropriately investigated and resolved. Experiments were performed under a project license (No.:2018-L062) granted by the Ethics Committee of the Affiliated Hospital of Nantong University, in compliance with GSPC (Gold standard publication checklist) for the care and use of animals.

Open Access Statement: This is an Open Access article distributed in accordance with the Creative Commons Attribution-NonCommercial-NoDerivs 4.0 International License (CC BY-NC-ND 4.0), which permits the noncommercial replication and distribution of the article with the strict proviso that no changes or edits are made and the original work is properly cited (including links to both the formal publication through the relevant DOI and the license). See: https://creativecommons.org/licenses/by-nc-nd/4.0/.

\section{References}

1. Martin GS, Mannino DM, Eaton S, et al. The epidemiology of sepsis in the United States from 1979 through 2000. N Engl J Med 2003;348:1546-54.

2. Quenot JP, Dargent A, Large A, et al. Treatment of sepsisinduced acute kidney injury in the ICU: the therapeutic targets do not seem to be established yet. Ann Transl Med 2019;7:S181.

3. de Pablo R, Monserrat J, Prieto A, et al. Role of circulating lymphocytes in patients with sepsis. Biomed Res Int 2014;2014:671087.

4. Wang Q, Zhou X, Yang L, et al. Gentiopicroside (GENT) protects against sepsis induced by lipopolysaccharide (LPS) through the NF- $\kappa \mathrm{B}$ signaling pathway. Ann Transl Med 2019;7:731.

5. Ma S, Evans RG, Iguchi N, et al. Sepsis-induced acute kidney injury: A disease of the microcirculation. Microcirculation 2019;26:e12483.

6. Xu W, Chen X, Tang Y. Study on the pathogenesis of acute kidney injury in sepsis. Journal of Community Medicine 2012;10:15-7.

7. Zarjou A, Agarwal A. Sepsis and acute kidney injury. J Am Soc Nephrol 2011;22:999-1006.

8. Federspiel CK, Itenov TS, Mehta K, et al. Duration of acute kidney injury in critically ill patients. Ann Intensive
Care 2018;8:30.

9. Moon SH, Jenkins CM, Liu X, et al. Activation of mitochondrial calcium-independent phospholipase A2 $\gamma($ iPLA2 $\gamma$ ) by divalent cations mediating arachidonate release and production of downstream eicosanoids. J Biol Chem 2012;287:14880-95.

10. Nevalainen TJ, Kortesuo PT, Rintala E, et al. Immunochemical detection of group I and group II phospholipases A2 in human serum. Clin Chem 1992;38:1824-9.

11. Kishimoto K, Matsumura K, Kataoka Y, et al. Localization of cytosolic phospholipase A2 messenger RNA mainly in neurons in the rat brain. Neuroscience 1999;92:1061-77.

12. Touqui L, Arbibe L. A role for phospholipase A2 in ARDS pathogenesis. Mol Med Today 1999;5:244-9.

13. Büchler $M$, Malfertheiner $P$, Schädlich $H$, et al. Role of phospholipase A2 in human acute pancreatitis. Gastroenterology 1989;97:1521-6.

14. Elliott TS, Casey AL, Karpanen TJ, et al. Addition of PLA2 to CRP enhances sepsis diagnosis. J Infect 2016;73:386-8.

15. Macphee CH. Lipoprotein-associated phospholipase A2: a potential new risk factor for coronary artery disease and a therapeutic target. Curr Opin Pharmacol 2001;1:121-5.

16. Carlquist JF, Muhlestein JB, Anderson JL. Lipoproteinassociated phospholipase A2: a new biomarker for cardiovascular risk assessment and potential therapeutic target. Expert Rev Mol Diagn 2007;7:511-7.

17. Gorelick PB. Lipoprotein-associated phospholipase A2 and risk of stroke. Am J Cardiol 2008;101:34F-40F.

18. Cai R, Huang R, Han J, et al. Lipoprotein-associated Phospholipase A2 Is Associated with Risk of Mild Cognitive Impairment in Chinese Patients with Type 2 Diabetes. Sci Rep 2017;7:12311.

19. Zalewski A, Macphee C, Nelson JJ. Lipoprotein-associated phospholipase A2: a potential therapeutic target for atherosclerosis. Curr Drug Targets Cardiovasc Haematol Disord 2005;5:527-32.

20. Gao Y, Zhao X, Wang S. Recent research progress of lipoprotein associated phospholipase A2. Chinese Journal of Arteriosclerosis 2008;16:922-4.

21. Simonini A, Moscucci M, Muller DWM, et al. IL-8 is an angiogenic factor in human coronary atherectomy tissue. Circulation 2000;101:1519-26.

22. De Stefano A, Mannucci L, Tamburi F, et al. LpPLA2, a new biomarker of vascular disorders in metabolic diseases. Int J Immunopathol Pharmacol 2019;33:2058738419827154. 
23. Li D, Wei W, Ran X, et al. Lipoprotein-associated phospholipase A2 and risks of coronary heart disease and ischemic stroke in the general population: A systematic review and meta-analysis. Clin Chim Acta 2017;471:38-45.

24. Bedirli A, Gokahmetoglu S, Sakrak O, et al. Beneficial effects of recombinant platelet-activating factor acetylhydrolase and BN 52021 on bacterial translocation in cerulein-induced pancreatitis. Eur Surg Res 2004;36:136-41.

25. Onyimba JA, Coronado MJ, Garton AE, et al. The innate immune response to coxsackievirus $\mathrm{B} 3$ predicts progression to cardiovascular disease and heart failure in male mice. Biol Sex Differ 2011;2:2.

26. Rittirsch D, Huber-Lang MS, Flierl MA, et al. Immunodesign of experimental sepsis by cecal ligation and puncture. Nat Protoc 2009;4:31-6.

27. Yang S, Chung CS, Ayala A, et al. Differential alterations in cardiovascular responses during the progression of polymicrobial sepsis in the mouse. Shock 2002;17:55-60.

Cite this article as: Liang $\mathrm{G}, \mathrm{Wu}$ R, Jiang L, Liu Y, Mao G, Huang Z, Qi L, Jiang H. The role of lipoprotein-associated phospholipase A2 in acute kidney injury of septic mice. Transl Androl Urol 2020;9(5):2192-2199. doi:10.21037/tau-20-1173
28. Leelahavanichkul A, Yasuda H, Doi K, et al. Methyl-2acetamidoacrylate, an ethyl pyruvate analog, decreases sepsis-induced acute kidney injury in mice. Am J Physiol Renal Physiol 2008;295:F1825-35.

29. Van Biesen W, Van Massenhove J, Lameire N, et al. Does urinary neutrophil gelatinase-associated lipocalin really solve the issue of discriminating prerenal from intrinsic acute kidney injury. Kidney Int 2012;81:321; author reply 321-2.

30. Stafforini DM, Sheller JR, Blackwell TS, et al. Release of free F2-isoprostanes from esterified phospholipids is catalyzed by intracellular and plasma platelet-activating factor acetylhydrolases. J Biol Chem 2006;281:4616-23.

31. Zalewski A, Macphee C. Role of lipoprotein-associated phospholipase A2 in atherosclerosis: biology, epidemiology, and possible therapeutic target. Arterioscler Thromb Vasc Biol 2005;25:923-31.

(English Language Editor: J. Gray) 